\title{
RAD50 loss of function variants in the zinc hook domain associated with higher risk of familial esophageal squamous cell carcinoma ${ }^{\dagger}$
}

\author{
Josephine Mun-Yee Ko ${ }^{1, \#, *}$, Shiu-Yeung Lam ${ }^{1, *}$, Lvwen Ning ${ }^{1}$, Annie Wai Yeeng Chai ${ }^{1}$, Lisa \\ Chan Lei ${ }^{1}$, Sheyne Sta Ana Choi ${ }^{1}$, Carissa Wing-Yan Wong ${ }^{1}$, and Maria Li Lung ${ }^{1, *}$ \\ 1 Department of Clinical Oncology, University of Hong Kong, Hong Kong (Special Administrative Region), \\ People's Republic of China; joko@hku.hk, mrlam510@gmail.com, u3003773@connect.hku.hk, \\ waiyeeng@connect.hku.hk, u3004461@connect.hku.hk, ssachoi2@connect.hku.hk, wwyc23@hku.hk, \\ mlilung@hku.hk \\ \# Co-first authors \\ * Correspondence: mlilung@hku.hk (M.L.L.); Tel.: 852-3917-9783 (M.L.L.); joko@hku.hk (J.M.Y.K.); Tel.: 852- \\ 3917-6931 (J.M.Y.K.) \\ + Presented at the 1 st International Electronic Conference on Cancers: Exploiting Cancer Vulnerability by \\ Targeting the DNA Damage Response (IECC 2021), online, and 01-14 February 2021.
}

Received: date; Accepted: date; Published: date

\begin{abstract}
Unbiased whole-exome sequencing approaches in familial ESCC initially prioritized RAD50 as a candidate cancer predisposition gene. The combined study with 3289 Henan individuals identified two pathogenic RAD50 protein truncation variants, p.Q672X and a recurrent p.K722fs variant at the zinc hook domain significantly conferring increased familial ESCC risk. Effects of $~ 10$-fold higher familial ESCC risk were observed, when compared to East Asians from the gnomAD database. Functional characterization suggests that the $R A D 50 \mathrm{Q} 672 \mathrm{X}$ mutation contributes a dominant-negative effect in DNA repair. Our study suggests RAD50 LOF variants in the zinc hook domain associate with a higher risk of familial ESCC in Chinese.
\end{abstract}

Keywords: Familial ESCC; RAD50; DNA repair, Loss of function mutation, NGS, Chinese, Genetic susceptibility, Zinc hook domain, ATM/ATR Synthetic lethality, CHK1 inhibitor

\section{Introduction}

Esophageal cancer (EC) is ranked 9th among cancers in global incidence with 572,034 newly diagnosed cases and a dismal 5-year survival rate of less than 5\% [1]. EC poses immense health risk worldwide [1]. The distribution of histological subtypes of EC displays geographical correlations. In Eastern countries where EC is prevalent, such as China, squamous cell carcinoma (SCC) is the predominant histological EC subtype, as opposed to adenocarcinoma (AC) being the more common form of EC in the West [2]. The epidemiological risk factors of esophageal squamous cell carcinoma (ESCC) differ between the high-risk China and moderate-risk Japanese regions [2]. Although GWAS studies provided genetic evidence for the modest effect of common variants and ESCC risk, the current understanding of the genetic pathogenesis and the genetic risk of rare deleterious variants with large effects on ESCC is still poor [3-5]. ESCC occurs with an extraordinarily high incidence with dismal survival in Northern China, highlighting the unmet need for early detection to improve patient survival. The management of the disease remains challenging in high-risk areas such as Henan, Shanxi and the Tai-Hang mountain area. Familial aggregation in the high-risk region implicates genetic factors as playing an etiological role [6, 7]. Hence, we performed a next-generation sequencing (NGS) study utilizing whole-exome sequencing (WES) and deep target capture sequencing of an oncology panel of 598 genes with ESCC patients from Henan, a hotspot of ESCC incidence in China, to understand the genetic basis for this cancer. Our earlier study identified 
multiple candidate cancer predisposition genes including genes involved in DNA repair (BRCA2, $P O L Q$, and MSH2), inflammation (TTC39B), and angiogenesis (KDR), significantly associated with ESCC risk [8]. RAD50 was prioritized by applying a loss-of-function (LOF) filtering strategy in our initial effort as one of the top candidate genes for further data mining to reveal its role in genetic susceptibility in ESCC development in the high-risk region.

RAD50 mapping to $5 \mathrm{q} 31$ encodes a $153 \mathrm{kDa}$ protein, which is a part of the MRE11-RAD50-NBS1 (MRN) complex. The multiprotein complex orchestrates the repair of DNA double-strand breaks (DSBs) by exerting its effect in various stages of cellular DNA damage response (DDR) including DSB sensing, DDR cascade initiation and repair pathway decisions to maintain genome stability [9-11]. Notably, the MRN complex is responsible for the activation of ataxia-telangiectasia mutated (ATM), the apical signalling kinase that initiates cell cycle arrest and the vast downstream network of effectors in DDR to DSBs [12, 13].

The involvement of RAD50 in ESCC is unknown, while it is one of the ten DNA repair genes critical for hereditary breast cancer [14]. Germline RAD50 mutations may contribute to hereditary cancer in human as hypomorphic RAD50 mutants exhibited cancer predisposition in mice [15]. Hence, the current study aimed to utilize NGS approaches to study the role of RAD50 in familial ESCC risk in the high-risk region from China. The current study showed by massive parallel sequencing that rare germline LOF variants of RAD50 (RAD50Q672X and RAD50K722fs) in the zinc hook domain were significantly elevated in familial ESCC cases with large effects.

The potential clinical implication of therapeutically targeting RAD50 in cancers has been demonstrated by dominant negative disruption of RAD50, conferring sensitization to platinumbased chemotherapy in squamous cell carcinoma [16]. Hence, we also aimed to perform functional characterization of RAD50 mutants with regards to DNA damage stimuli inducing DSBs and various genotoxic agents. Our results suggest the dominant negative effects of the RAD50Q672X mutant impaired cellular responses to DSB repair and enhanced sensitivity to genotoxic agents including checkpoint inhibitor AZD7762 and formaldehyde-induced replication stress. Our study demonstrates the potential clinical utility of screening RAD50 mutations for early detection and provide therapeutic insights to benefit the familial ESCC patients.

\section{Materials and Methods}

The current study included a total of 3289 participants (1044 familial and 1074 sporadic ESCC, and 1171 controls) collected during 2001-2014 from Northern China high-risk Linxian and Anyang counties from Linzhou Center Hospital and Yaocun Esophageal Cancer Hospital provided by Lidong Wang (Zhengzhou University) for an earlier study[8]. Approval for use of human blood and/or information was obtained from the Committee for Ethical Review of Research Involving Human Subjects at Zhengzhou University. The study was conducted according to the Declaration of Helsinki principles. Informed written consent was obtained from all participants.

\section{WES, target capture sequencing and bioinformatics analysis}

Blood DNAs were extracted by QIAamp DNA Blood Mini Kit (QIAGEN, Germany). Details of library preparation with the KAPA HTP Library Prep Kit, exome sequencing and target capture with NimbleGen SeqCap EZ capture kits (Roche, Switzerland), were as previously published [8]. The data from 3289 individuals were processed using the analysis pipeline following the GATK guideline, as previously described $[8,17]$. Briefly, raw fastq reads were cleaned and then mapped to the human reference genome hg19 using the Burrows-Wheeler Aligner (BWA). PCR duplicates are marked using Picard. InDels realignment and variants recalibration were performed using GATK. ANNOVAR was used for functional annotation of variants. Combined annotation dependent depletion (CADD) score was used to assess damage effects of variants of unknown significance [18]. The rare variants of unknown significance with CADD score of $\geq 25$ (version1.4) are considered deleterious variants and listed in supplementary Table S1.

\section{Statistical analysis}


In NGS analysis, for the rare RAD50 LOF variant, the Fisher's exact test (two-tailed) was used for calculation of the Odds Ratio (OR) between cases and control using a Python package SciPy and a $p<0.05$ was considered statistically significant. Student's t test was used in statistical analyses in the cell viability and colony formation assays. A $p$ value $<0.05$ was considered as statistically significant. The error bars in the figures represent the standard error mean.

\section{Cell culture}

KYSE150, an ESCC cell line derived from poorly differentiated primary tumor, and U2OS, an osteosarcoma cell line, were used in the in vitro studies. KYSE150 was cultured with RPMI medium as previously described [19]. U2OS was cultured using Dulbecco's modified Eagle's medium (DMEM) with 10\% Fetal Bovine Serum and 1\% penicillin and streptomycin.

\section{RAD50 constructs, lentiviral preparation and transduction}

Functional knockout of human $R A D 50$ and expression of $R A D 50^{\mathrm{WT}}$ and mutants were performed via lentiviral transduction using the LentiCRISPRv2 plasmid, as previously described [20, 21]. Functional knockout of RAD50 in cell lines was performed with the sgRNA sequences: LacZ FwdCTCTGGCTAACGGTACGCGTA, Rev-TACGCGTACCGTTAGCCAGAG; S1GTTCCGCGTTACATAACTTA, Rev-TAAGTTATGTAACGCGGAAC as control, and ex2 (RAD50 KO) Fwd-CACCGTACATTTGTACACGATCCCA, Rev-AAACTGGGATCGTGTACAAATGTAC; ex10 (RAD50 KO $) \quad$ Fwd-CACCGCTAGGAACGTGAGTTAAGCA, RevAAACTGCTTAACTCACGTTCCTAGC as RAD50 KO sgRNAs. The subcloning of respective sgRNAs into the plasmid was performed as previously described. Expression of RAD50 wildtype and mutant constructs was performed using the pLVX-EF1a-Puro plasmid. Full-length CDS of RAD50 was isolated from pTP11 (a gift from Prof. Tanya Paull, University of Texas at Austin) and subcloned into pLVX-EF1a-Puro. Mutants of RAD50 were generated by the GeneArt ${ }^{\circledR}$ Site-directed Mutagenesis Kit (Invitrogen). Mutagenesis primers used are as follows: L1264F FwdCAGCGTAACTTCCAGTTTCTGGTAATCACTC, GAGTGATTACCAGAAACTGGAAGTTACGCTG, and Q672X FwdTCCCAGTTCATTACTTAGCTAACAGACGAAA, RevTTTCGTCTGTTAGCTAAGTAATGAACTGGGA.

\section{Western blotting}

Western blots were performed with primary antibodies anti-RAD50 (GTX70228, Genetex), antiMRE11 (GTX70212, Genetex), anti-NBS1 (GTX70224, Genetex), anti-6xHIS (\#12698, Cell Signaling Technology), anti- $\alpha$-tubulin (GTX112141, Genetex), and anti-P84 (GTX70220, Genetex), as described previously [20, 21].

\section{Ionizing radiation and immunofluorescence staining}

Cells received the ionizing radiation treatment at a cumulative dose of 10Gy by Gamma Irradiator MDS Gammacell 3000 Elan. Immunofluorescence staining was performed as previously described [21]. Briefly, cells were fixed with $4 \%$ paraformaldehyde and permeabilized with $0.1 \%$ Triton X-100 in PBS. Primary antibody anti- $\gamma$ H2AX (\#9718, Cell Signaling Technology) was incubated overnight at $4^{\circ} \mathrm{C}$ and with Alexa-488 Fluor ${ }^{\circledR}$ secondary antibodies for 1 hour at room temperature, in dark. Cells were visualized using Nikon Ti2-E Widefield Imaging System and image analyzed with Image J. For foci quantification, cells with $>10$ foci were counted. At least 1000 cells were counted for each sample.

\section{Cell viability and colony formation assays}

Cell viability was measured by the 3-(4,5-dimethylthiazol-2-yl)-2,5-diphenyl-tetrazolium bromide (MTT) assay, as reported previously [22]. Briefly, $1 \times 10^{4}-2.5 \times 10^{4}$ cells were seeded in triplicates in each well of a 96-well plate. Cells were treated for cisplatin at concentration range from 10 to $1000 \mathrm{nM}$, formaldehyde at concentration range from $10-800 \mu \mathrm{M}$, and AZD7762 at concentration range from 5 - $100 \mathrm{nM}$. Colony formation assay was performed as previously described [22]. Briefly, fixation and staining with Giemsa, colonies were counted in DMSO and AZD7762 (20 nM) treatment after one week. All experiments were repeated twice. 


\section{Results}

\section{WES analysis prioritizes RAD50 as top candidate CPG for familial ESCC}

By combining the enrichment of genetic component strategy and unbiased WES approach, we performed NGS analysis for 186 familial ESCC individuals with two generations and $\geq 2$ family members diagnosed with ESCC including proband [8]. After WES data analysis in the discovery phase applied a LOF filtering strategy, RAD50 was prioritized as the top candidate CPG for validation in a larger cohort (Table 1). The prevalence of RAD50 LOF variants in Henan family history-positive $(\mathrm{FH}+)$ ESCC patients $(4 / 372,1.1 \%)$ was significantly more frequent compared to that from the East Asian population in the gnomAD public database $(30 / 19,954,0.15 \%)(\mathrm{OR}=7.22, p=0.0033)$. Two out of the four RAD50 LOF variants (c.C2014T:p.Q672X and c.2165_2166insT:p.K722fs) are located at the zinc hook domain at amino acids 635-734 [23].

Two RAD50 LOF variants at the zinc hook domain associate with increased risk of familial ESCC

We performed target whole RAD50 sequencing in a larger cohort of 3103 ESCC patients including additional 858 familial ESCC (defined as $\geq 2$ family members diagnosis with ESCC including proband) and 1074 sporadic ESCC, and 1171 controls. For the validation cohort, we detected 3/1716 (0.17\%) RAD50 LOF variants in familial ESCC, 4/2148 (0.19\%) in sporadic ESCC and $4 / 2342(0.17 \%)$ in the controls (Table 1$)$. Overall, a total of seven pathogenic RAD50 LOF variants were detected at exons 8, 13, 17, 19, and 23 (Figure 1) in 15 individuals. Figure 1 shows the lollipop schematic diagram of seven RAD50 LOF variants. Four of the fifteen carriers $(26.7 \%, 4 / 15)$ had LOF $R A D 50$ variants mapping to exon 13 containing the zinc hook domain. Since the frequency of $R A D 50$ LOF mutations are similar between sporadic ESCC and controls, the sporadic ESCC and control cohorts were combined to estimate the risk of familial ESCC patients. In the familial ESCC patients, the frequency of two pathogenic variants, p.Q672X and the other recurrent p.K722fs variant (4/2088, $0.19 \%)$ are statistically higher than that in the sporadic ESCC and controls (0/4490, 0\%) (OR inf, $p=$ 0.010) (Table 2). An increased risk with large effect in familial ESCC patients carrying these two pathogenic $R A D 50 \mathrm{LOF}$ variants is also observed, when compared to East Asians from the gnomAD database $(4 / 19,954,0.02 \%)$ (OR 9.57, $\left.p=4.1 \times 10^{-3}\right)$ and all populations from gnomAD (5/282,670, $0.0018 \%$ ) (OR 108.51, $\left.p=3.5 \times 10^{-7}\right)$. p.Q672X is a very rare truncating $R A D 50$ mutation disrupting the zinc hook and confers a very high familial ESCC risk of more than 100 -fold $(0.045 \%, 1 / 2088)$ compared to all populations from genomAD (MAF 0.00035\%, 1/282,670) (OR 135.44, $p=0.015$ ). We provide further functional characterization and suggest that this variant contributes a dominant negative effect in DNA repair. The recurrent p.K722fs was more frequent in familial ESCC (3/2088, 0.14\%) compared to sporadic ESCC and control (0/4490,0\%) (OR inf, $p=0.032$, Table 2). p.K722fs only occurs in the East Asian population of gnomAD with minor allele frequency (MAF) $0.02 \%(4 / 19,945)(\mathrm{OR}=$ $7.18, p=0.022)$, but is absent in other populations of gnomAD including African, Latino, Jewish, European, and South Asian (0.0014\%, 4/282,670) (OR 101.73, $\left.p=1.3 \times 10^{-5}\right)$.

The estimated risk of familial ESCC carrying LOF RAD50 variants $(7 / 2088,0.34 \%)$ was moderately increased compared to Henan sporadic ESCC cases and controls (8/4490, 0.18\%), although this was not statistically significant (OR 1.88, $p=0.33$ ). A similar trend of moderately increased risk $(30 / 19,945,0.15 \%)(\mathrm{OR} 2.22, p=0.092)$ was observed, when compared to East Asians from the gnomAD database and all populations from gnomAD (427/282,670,0.17\%) (OR 2.22, $p=$ 0.062, Table 2).

\section{Sanger sequencing validation of RAD50 germline variants}

All individuals carrying RAD50 LOF variants (Table 1) and deleterious missense variants with CADD score $\geq 25$ present in familial ESCC including c.G2177A:p.R726H, c.C2287T:p.R763C, c.G3716A:p.R1239Q, and c.C3790T:p.L1264F (Table S1), were validated by Sanger sequencing, as shown in Figure 2 for representative validation of LOF variants c.C2014T:p.Q672X, and c.2165_2166insT:p.K722fs located at exon 13 containing the zinc hook domain and the missense c.C3790T:p.L1264F variant at exon 25. 
Table 1. : RAD50 LOF mutations identified in familial Chinese ESCC patients, sporadic ESCC and controls in 3289 individuals from Henan

\begin{tabular}{|c|c|c|c|c|c|c|c|c|c|}
\hline Chr5 (hg19) & Mutation (DNA) & Exon & Protein change & $\begin{array}{c}\text { FH+ cases } \\
\text { Discovery } \\
(n=372) \\
\text { Validation } \\
(n=1716)\end{array}$ & $\begin{array}{c}\text { Sporadic cases } \\
\text { Validation } \\
(\mathrm{n}=\mathbf{2 1 4 8})\end{array}$ & $\begin{array}{c}\text { Controls } \\
\text { Validation } \\
(\mathrm{n}=\mathbf{2 3 4 2 )}\end{array}$ & $\begin{array}{l}{\text { gnom } A D^{b}}^{b} \\
\text { East Asian } \\
(n=19954)\end{array}$ & $p$ & OR \\
\hline
\end{tabular}

Whole-exome sequencing of discovery cohort of 186 familial ESCC involved two generations

\begin{tabular}{|c|c|c|c|c|c|c|c|c|c|}
\hline 131931309 & c.C2014T & 13 & p.Q672X & 1 & NA & NA & 0 & \multirow{4}{*}{$0.0033^{c}$} & \multirow{4}{*}{$7.22^{\circ}$} \\
\hline 131931460 & c.2165_2166insT & 13 & p.K722fs & 1 & NA & NA & 4 & & \\
\hline 131945032 & c.2980_2983del & 19 & p.K994fs & 1 & NA & NA & 8 & & \\
\hline 131951735 & c.3077_3080del & 20 & p.K1026fs & 1 & NA & NA & Not reported & & \\
\hline
\end{tabular}

Target whole-gene sequencing of RAD50 in validation cohort of 3103 individuals containing 858 familial and 1074 sporadic ESCC and 1171 controls

\begin{tabular}{|c|c|c|c|c|c|c|c|c|c|}
\hline 131924437 & c.1110delA & 8 & p.L370fs & 1 & 2 & 2 & Not reportedg & $0.37^{\mathrm{d}}$ & $1.97^{\mathrm{d}}$ \\
\hline 131931460 & c.2165_2166insT & 13 & p.K722fs & 2 & 0 & 0 & 4 & \multirow{3}{*}{$0.38^{\mathrm{e}}$} & \multirow{3}{*}{1.80} \\
\hline 131944319 & c.C2731T & 17 & p.Q911X & 0 & 0 & 1 & Not reported & & \\
\hline 131945032 & c.2980_2983del & 19 & p.K994fs & 0 & 1 & 1 & 8 & & \\
\hline 131973850 & c.C3553T & 23 & p.R1185X & 0 & 1 & 0 & 1 & $1.00^{\mathrm{f}}$ & $1.09^{\mathrm{f}}$ \\
\hline
\end{tabular}

aNucleotide position are based on the NM 005732 transcript of RAD50. ${ }^{\mathrm{F} F r e q u e n c i e s}$ of RAD50 variants are exported from gnomAD, https://gnomad.broadinstitute.org/ on June 5, 2020.

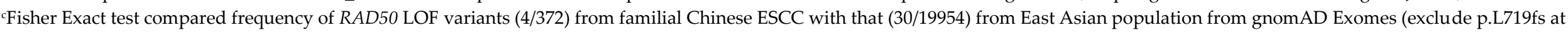
chr5: 131931451) in the discovery cohort. $n=$ number of allleles

dFisher Exact test compared frequency of RAD50 LOF gain variants (7/2088) from familial Chinese ESCC with that (4/2342) from Henan controls combining discovery and validation cohorts. eFisher Exact test compared frequency of RAD50 LOF variants (7/2088) from familial Chinese ESCC with that (4/2148) from sporadic ESCC combining discovery and validation cohorts. fFisher Exact test compared frequency of RAD50 LOF variants (4/2148) from sporadic ESCC with that (4/2342) from controls.

sp.L370fs reported with frequency of 1/15314 in a RAD50 association study for Chinese breast cancer patients (n=7657) [24]. 
Table 2. : Estimated risk of familial ESCC with LOF RAD50 variants in Henan cohort of familial ESCC versus sporadic ESCC and controls, and East Asian population or all populations from gnomAD.

\begin{tabular}{|c|c|c|c|c|c|c|c|c|c|c|c|}
\hline Mutation & $\begin{array}{c}\text { FH+ } \\
\text { ESCC } \\
(n=2,088)\end{array}$ & $\begin{array}{c}\text { Sporadic } \\
\text { ESCC } \\
(n=2,148)\end{array}$ & $\begin{array}{l}\text { Controls } \\
(n=2,342)\end{array}$ & $p^{a}$ & $\mathrm{OR}^{\mathrm{a}}$ & $\begin{array}{l}{\text { gnom } A D^{d}} \\
\text { East Asian } \\
(n=19,954)\end{array}$ & $p^{b}$ & $\mathrm{OR}^{\mathrm{b}}$ & $\begin{array}{c}\text { gnomAD } \\
\text { All } \\
(n=282,670)\end{array}$ & $p^{c}$ & $\mathrm{OR}^{\mathrm{c}}$ \\
\hline p.Q672X & $0.045 \%(1)$ & $0 \%$ & $0 \%$ & 0.32 & $\inf$ & $0 \%$ & 0.095 & Inf & $0.00035 \%(1)$ & 0.015 & 135.44 \\
\hline p.K722fs & $0.14 \%(3)$ & $0 \%$ & $0 \%$ & 0.032 & $\inf$ & $0.02 \%(4)$ & 0.022 & 7.18 & $0.0014 \%(4)$ & $1.3 \times 10^{-5}$ & 101.73 \\
\hline p.Q672X/p.K722fs & $0.19 \%(4)$ & $0 \%$ & $0 \%$ & 0.010 & $\inf$ & $0.02 \%(4)$ & $4.1 \times 10^{-3}$ & 9.57 & $0.0018 \%(5)$ & $3.5 \times 10^{-7}$ & 108.51 \\
\hline All RAD50 LOF & $0.34 \%(7)$ & $0.19 \%(4)$ & $0.17 \%(4)$ & 0.33 & 1.88 & $0.15 \%(30)$ & 0.092 & 2.23 & $0.17 \%(427)$ & 0.062 & 2.22 \\
\hline
\end{tabular}

${ }^{a} p$ value and OR of Fisher Exact test comparing frequency of RAD50 zinc hook mutations from Henan Chinese familial ESCC with that from sporadic ESCC and controls (0/4490). pL719fs was filtered.

${ }^{b} p$ value and OR of Fisher Exact test comparing frequency of RAD50 zinc hook mutations from Henan Chinese familial ESCC with that from East Asian population from gnomAD.

${ }^{c} p$ value and OR of Fisher Exact test comparing frequency of RAD50 zinc hook mutations from Henan Chinese familial ESCC with that from all populations from gnomAD.

'Frequencies of RAD50 variants are exported from gnomAD, https://gnomad.broadinstitute.org/ on June 5, 2020. 


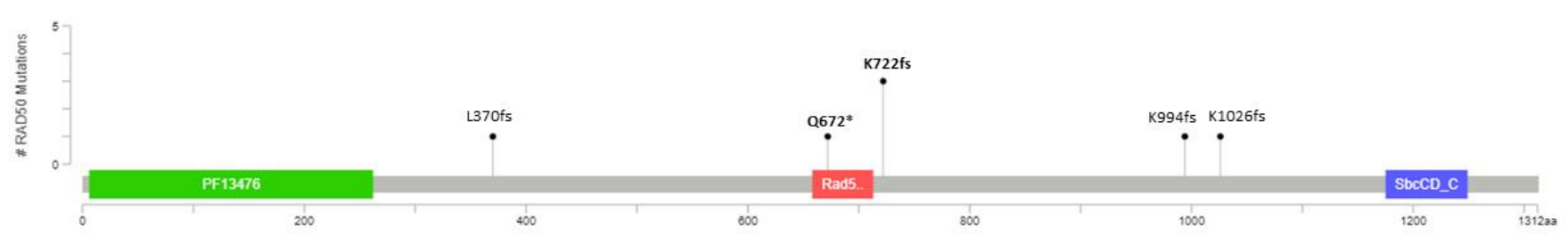

(a)

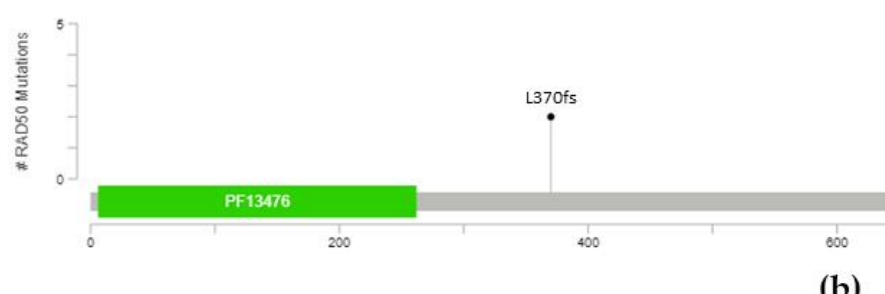

(b)

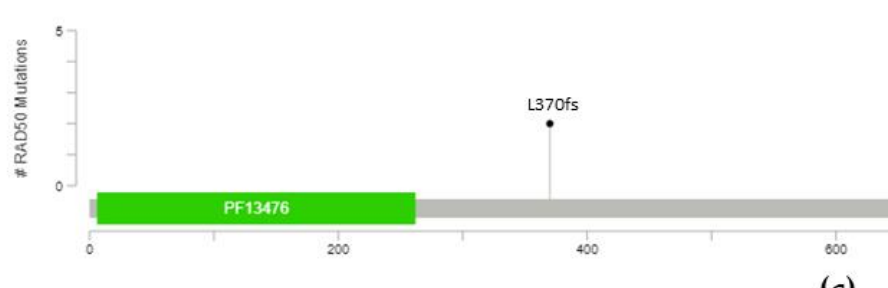

(c)

Figure 1. Lollipop schematic diagram of RAD50 LOF mutation distribution in (a) familial ESCC cases, (b) sporadic ESCC cases, and (c) controls from Henan.

RAD50:uc003kxi.3:exon13:c.C2014T:p.Q672X

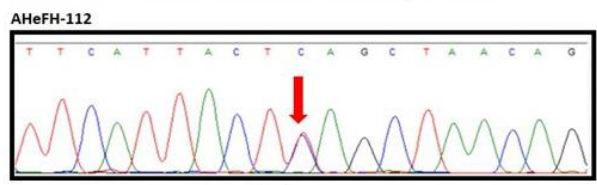

(a)

RAD50:uc003kxi.3:exon25:c.C3790T:p.L1264F

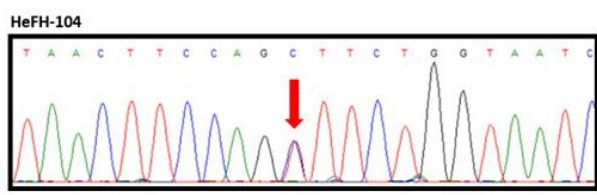

(c)
RAD50:uc003kxi.3:exon13:c.2165_2166insT:p.K722fs AHeFH-0596

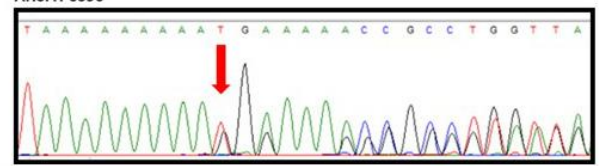

AHeFH-2198

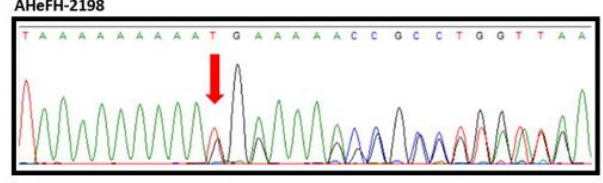

AHeFH_0017

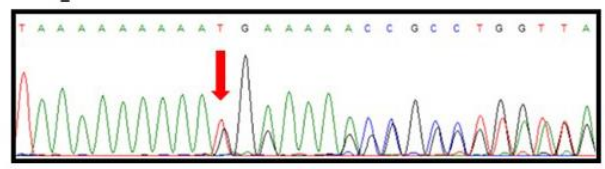

(b)

Figure 2. Sanger sequencing validation of RAD50 LOF germline mutations, (a) c.C2014T:p.Q672X, (b) c.2165_2166insT:p.K722fs, and (c) c.C3790T:p.L1264F in five familial ESCC patients. 


\section{$R A D 50$ is indispensable for the survival of ESCC cells}

As a gene well-documented to be essential to cellular survival, RAD50 protein is constitutively expressed in a panel of fifteen ESCC cell lines (Figure 3a). The expressions of RAD50 in ESCC cell lines KYSE150 and KYSE180TS were the highest amongst the ESCC cell lines tested. When compared to NE1, an immortalized cell line derived from normal esophageal tissue, ESCC cell lines generally showed reduced RAD50 expression levels. To study the functional role of RAD50 in ESCC, we performed CRISPR-Cas9 mediated functional knockout of RAD50 in ESCC cell line KYSE150. After confirmation of successful depletion of RAD50 by Western blot (Figure 3b), it is observed that KYSE150 cells lost viability rapidly and ceased to proliferate, in contrast to controls in the knockout experiment. Such an observation conforms with the previous studies that revealed the essential role of $R A D 50$ in cellular survival.

\section{Dominant negative over-expression of $R A D 50^{0672 \mathrm{X}}$ mutant delays the repair of IR-induced DSBs}

From our NGS data, LOF and missense germline mutations of RAD50 that are potentially functionally disruptive were identified (Figure 1 and Table S1). In particular, mutant $R A D 50^{\mathrm{L} 1264 \mathrm{~F}}$ and $R A D 50 \mathrm{Q}^{672 \mathrm{X}}$ reside in close proximity to the ATP-binding cassette and zinc hook domain of RAD50, respectively. Hence, we performed site-directed mutagenesis to obtain the mutated constructs and overexpressed these mutants ( $\left.R A D 50^{\mathrm{L} 1264 \mathrm{~F}}, R A D 50^{\mathrm{Q} 672 \mathrm{X}}\right)$ and wildtype $R A D 50\left(R A D 50^{\mathrm{WT}}\right)$ in vitro to study the phenotypic consequences of these mutations. Upon validation of overexpression of $R A D 50$ constructs in both KYSE150 and U2OS by Western blot (Figure 3c), we examined the expression levels of the interacting proteins of RAD50 in the MRN complex. Overexpression of RAD50 ${ }^{\mathrm{WT}}$, RAD50 $1264 \mathrm{~F}$ and $R A D 50{ }^{6} 62 \mathrm{x}$ result in a proportional increase in the protein expression levels of MRE11 and NBS1 (Figure 3d).

The primary function of RAD50 or the MRN complex is in the initiation of DNA damage response to DSBs. Hence, we assessed the effect of mutant expression on the formation of $\gamma \mathrm{H} 2 \mathrm{AX}$ foci after irradiating cells with gamma-radiation for 30 minutes followed by 6 and 12 hours of recovery post-irradiation (Figure 3e). The overexpression of both RAD50 formation of $\gamma \mathrm{H} 2 \mathrm{AX}$ foci 30 minutes post-IR. Six hours after the initial IR treatment, the overexpression of RAD50WT showed more rapid repair of IR-induced DSBs, marked by the significantly reduced percentage of $\gamma \mathrm{H} 2 \mathrm{AX}$ foci-positive cells. Whilst overexpression of either $R A D 50^{\mathrm{L} 1264 \mathrm{~F}}$ or $R A D 50^{\mathrm{Q} 672 \mathrm{X}}$ did not enhance recovery of $\gamma \mathrm{H} 2 \mathrm{AX}$ foci, $R A D 50 \mathrm{Q} 672 \mathrm{X}$ showed further hindrance in the recovery of foci, as a significantly larger percentage of foci-positive cells persisted 6 hours and 12 hours post-IR.

\section{Dominant negative over-expression of RAD50 mutants sensitize cells to formaldehyde and CHK1 inhibitor AZD7762 treatment}

Previous studies targeting RAD50 or the MRN complex combined with cisplatin treatment resulted in tumor cell sensitization [16, 25, 26]. The dominant-negative functional impact of $R A D 500^{672 X}$ in delayed DSB repair upon IR treatment led us to test the sensitivity of cells expressing RAD50 wildtype and mutant constructs with cisplatin and a PARP inhibitor (PARPi). The MTT assay indicated that expression of RAD50 mutants did not potentiate cells towards cisplatin (Figure 4a) and PARPi treatment (Table S2). The dominant-negative LOF RAD50 mutant did not enhance therapeutic efficacy of platinum-based drugs. Previous study revealed RAD50 activated ATR signaling upon replication stress [27]. We tested the sensitivity of cells expressing RAD50 wildtype and mutant constructs towards formaldehyde that induces DNA damage through replication stress. The MTT results showed that $R A D 50^{\mathrm{L} 1264 \mathrm{~F}}$ and $R A D 50^{\mathrm{Q} 672 \mathrm{X}}$ cells were sensitized upon formaldehyde treatment at $100 \mu \mathrm{M}$ and towards AZD7762, a CHK1 inhibitor, with an IC50 of $40.72 \mathrm{nM}$ and $28.91 \mathrm{nM}$, respectively, compared to VA control $(64.53 \mathrm{nM})$ and $R A D 50^{\mathrm{WT}}$ (66.49 $\mathrm{nM}$ ) (Figure 4a). The sensitization towards AZD7762 treatment was also evidenced in the colony formation assay (Figure $4 \mathrm{~b})$. As for the phenotypic cause behind the observed sensitization, we observed, upon AZD7762 treatment, a significantly elevated percentage of $R A D 50^{L 1264 \mathrm{~F}}$ and $R A D 50^{\mathrm{Q} 672 \mathrm{X}}$ cells showed pannuclear $\gamma \mathrm{H} 2 \mathrm{AX}$ patterns, indicative of cytotoxic level of replication stress, in contrast to the vectoralone control and $R A D 50^{\mathrm{WT}}$ (Figure 4c). 


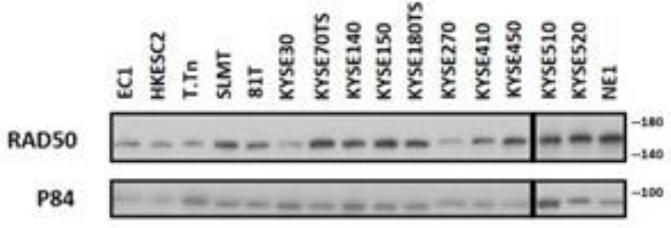

(a)

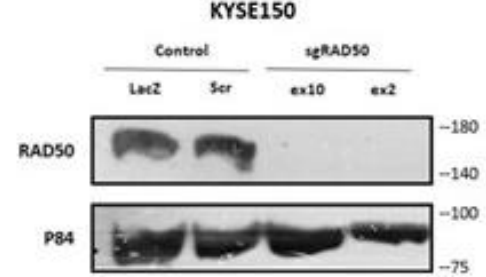

(b)

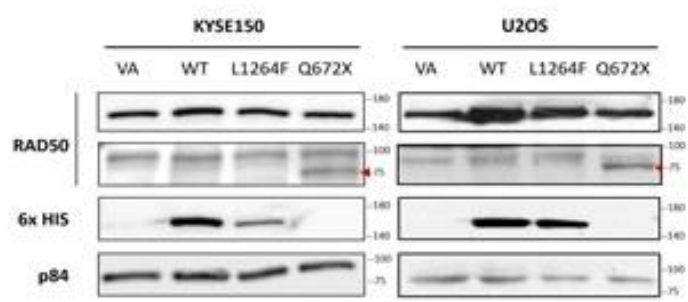

(c)

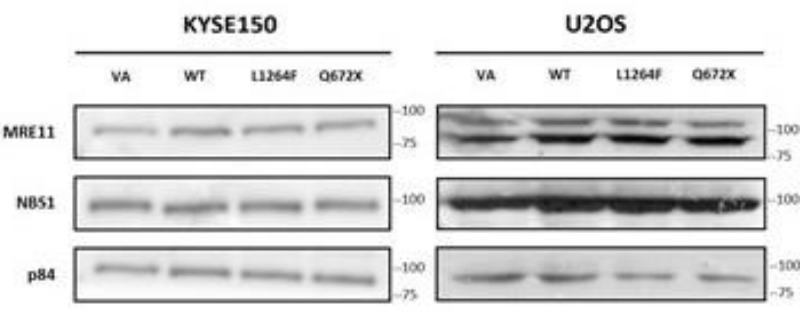

(d)

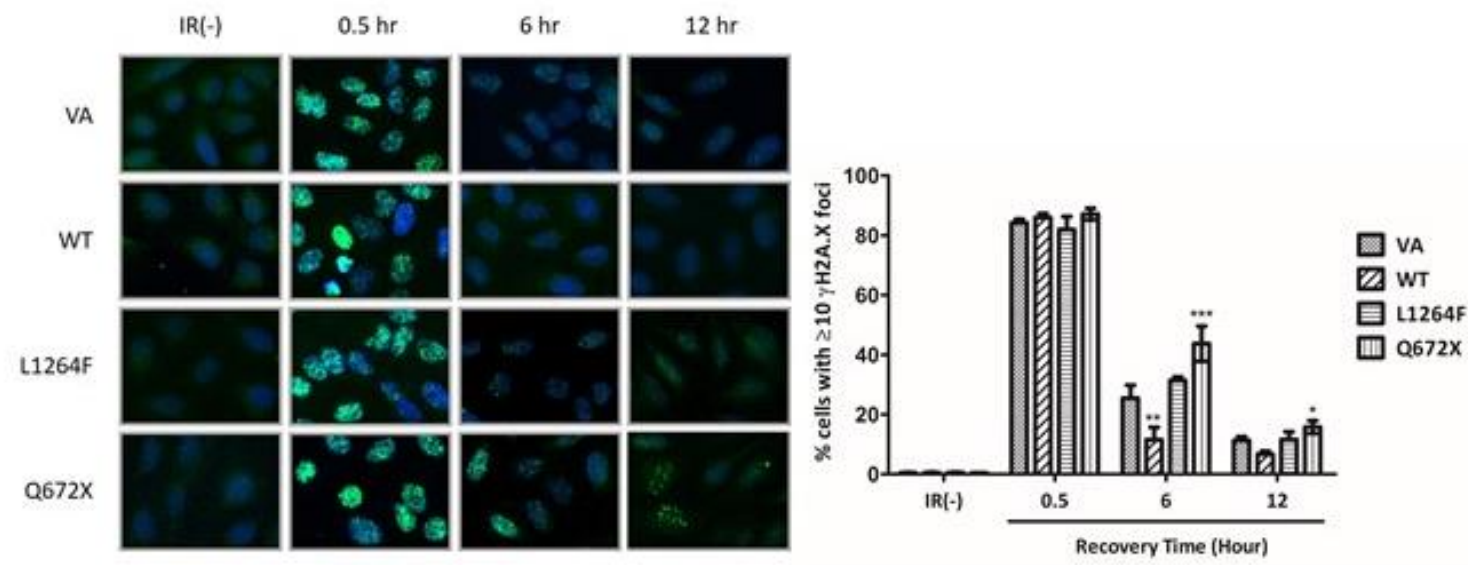

(e)

Figure 3. Dominant negative effect of DSB repair by over-expression of zinc hook RAD5 Q672X mutant. RAD50 ubiquitously expressed in ESCC cell lines (a); knock down of RAD50 in KYSE150 (b); overexpression of RAD50 mutants in KYSE150 and U2OS (c) induced higher expression of MRE11 in KYSE150 and MRE11/NBS1 in U2OS (d); delay of DSB repair ability indicated by $\gamma \mathrm{H} 2 \mathrm{AX}$ foci formation in $R A D 50^{\mathrm{Q} 672 \mathrm{X}}$ over-expression in U2OS after IR stimulation (e). 

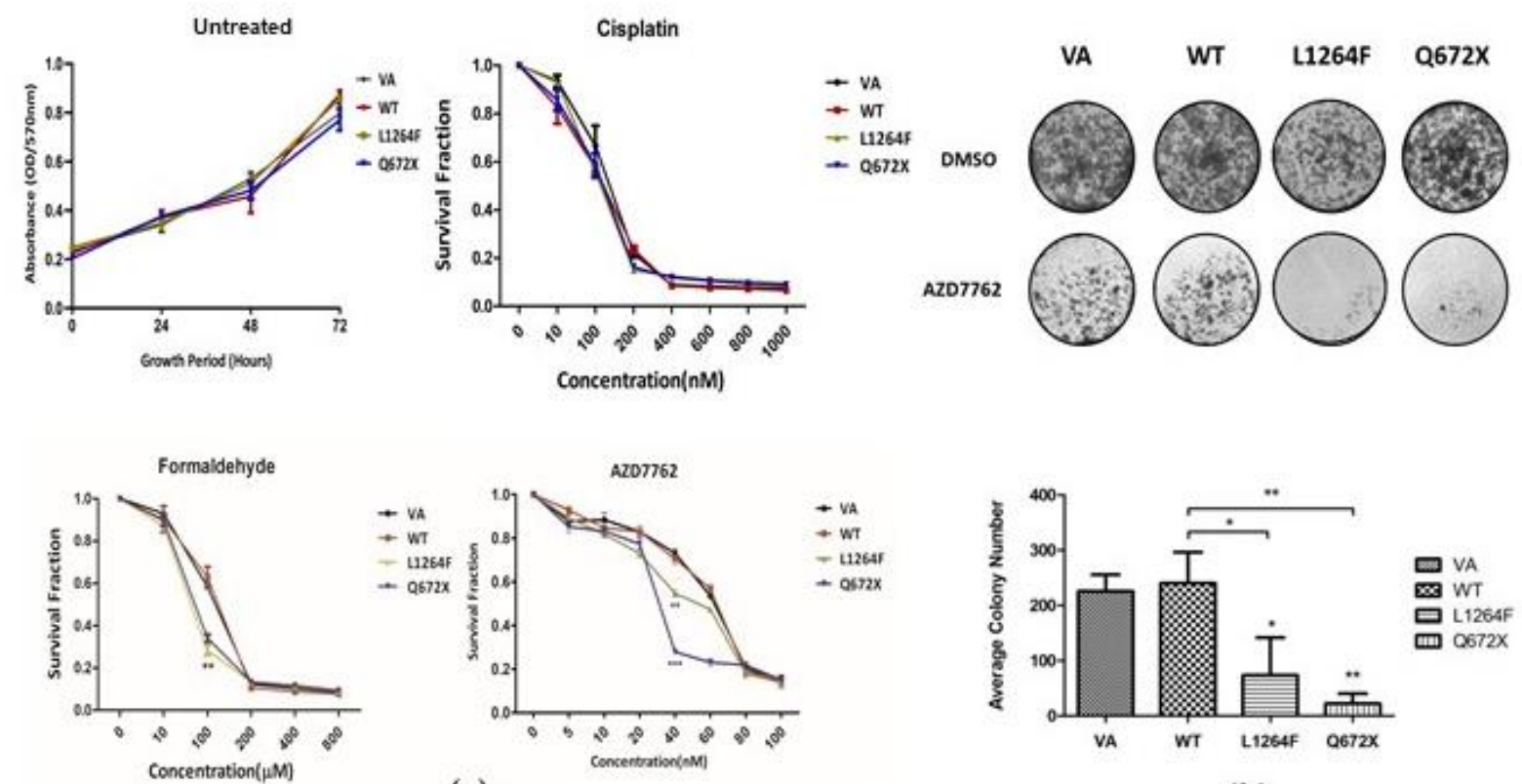

(a)

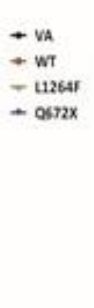

+ wT

$\sin 2 x$
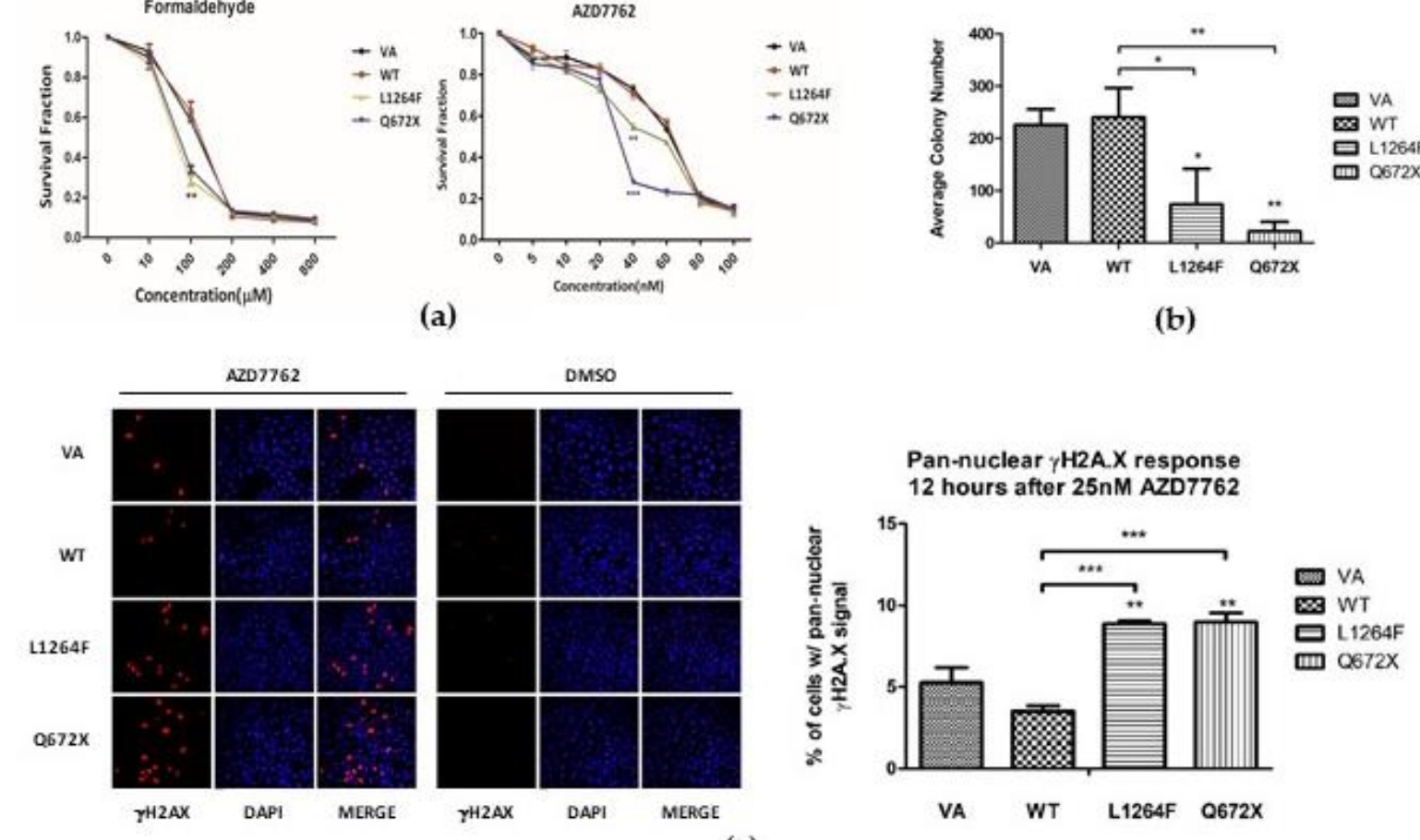

(b)

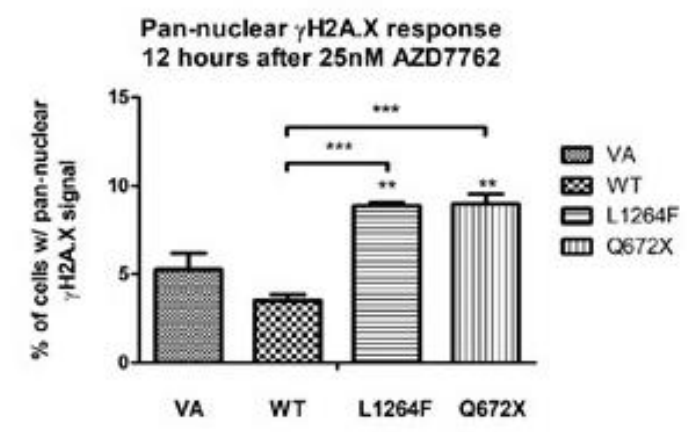

(c)

Figure 4. Dominant negative effect of over-expression of $R A D 50^{\mathrm{Q} 672 \mathrm{X}}$ and $R A D 50^{\mathrm{L} 1264 \mathrm{~F}}$ in U2OS sensitized cell viability to AZD7762 (CHK1 inhibitor) and formaldehyde treatment but not to cisplatin; (b) inhibited colony forming ability; (c) increased pan-nuclear $\gamma \mathrm{H} 2 \mathrm{~A}$.X response after AZD7762 treatment. 


\section{Discussion}

The genetic predisposition role of RAD50 in inherited breast cancer [14] has been documented, while its role in breast cancer is still controversial $[23,24,28]$. However, the genetic susceptibility role of RAD50 in familial ESCC is largely unknown. Our current NGS study is the first comprehensive investigation of the entire RAD50, (also includes MRE11A and NBS1) by hybridization-based target capture approach for the genetic predisposition role of the evolutionarily conserved MRN complex in the high-risk ESCC region. Our earlier publication utilized a gene-based association test and revealed no significant association of elevated cancer risk in Henan familial ESCC patients carrying LOF variants in RAD50-MRE11A-NBS1.[8] With more detailed data analysis in the RAD50 gene, two rare pathogenic LOF variants in the zinc hook domain of RAD50 were identified only present in familial ESCC patients, but was not found in the sporadic ESCC patients and controls (Table 2). These variants were about 100 -fold less frequent in all populations in the genomAD database. The $R A D 50 \mathrm{Q} 672 \mathrm{X}$ and $R A D 50^{\mathrm{K} 722 \mathrm{fs}}$ were not observed in another large NGS breast cancer study reporting a poor prognostic role of pathogenic RAD50 protein truncations in 7657 Chinese patients [24]. In this current study, the lack of survival data precluded assessing the prognostic role of pathogenic LOF RAD50 mutations in ESCC. Future NGS studies focusing on RAD50 germline or somatic mutations should address this issue.

Platinum-based drugs, such as cisplatin, are the common first-line chemotherapeutic drugs for ESCC. ESCC patients carrying dominant-negative germline or somatic RAD50 alterations with disrupted MRN function may have improved prognosis, as tumor cells are sensitized to cisplatin cytotoxicity. The increased DNA repair and telomere maintenance response by MRN may lead to chemoresistance. A dual disruption approach targets DNA repair and telomere maintenance to treat $B R C A$-proficient head and neck cancer by combining PARPi and dominant negative Nbs1 disruption of MRN [26]. However, the protein truncating RAD50Q672X mutant affecting the zinc hook and eliminating the C-terminal coiled-coil and ATPase domains resulted in a delay of DSB repair did not sensitize cells upon cisplatin or PARPi treatment. The dominant negative LOF RAD50 mutant did not enhance therapeutic efficacy of platinum-based drugs.

Tumors harboring a RAD50 ${ }^{\mathrm{L} 123 \mathrm{~F}}$ hypomorphic mutant allele at the D-loop motif of the RAD50 protein and defective ATM signaling demonstrated an outlier curative response in a recurrent patient treated with irinotecan and AZD7762, targeting CHK1 inhibition [29]. Mutations of the MRN complex were present in about $4 \%$ of all human tumors and clustered near the D-loop motif in various tumor types [29]. The synthetic lethal approach targeting ATM/CDK1 may sensitize cancer cells carrying defective ATM activity to cell death, as RAD50 mutant cells are strongly dependent on the $A T R$ checkpoint pathway for survival upon replication stress stimuli. Although the deleterious mutant $R A D 50^{\mathrm{L} 1264 \mathrm{~F}}$ did not associate with higher familial ESCC risk, our functional study suggested patients carrying heterozygous $R A D 50^{\mathrm{L} 1264 \mathrm{~F}}$ may have a dominant negative effect upon induction of replication stress by formaldehye treatment and the CHK1 inhibitor, AZD7762. Further study is necessary to investigate the potential therapeutic options for East Asian-specific $R A D 50^{\mathrm{L} 1264 \mathrm{~F}}$ variants (Table S1). The zinc hook domain of RAD50 is important for DNA repair [30]. Functional characterization of the zinc hook protein truncation mutant, $R A D 50 \mathrm{Q} 672 \mathrm{x}$, suggested a dominant negative effect in DSB repair and replication stress. The functional study further reinforced our NGS association of the elevated risk of germline LOF of RAD50 in the zinc hook domain with familial ESCC. Our data suggested that RAD50 zinc hook domain LOF mutation screening in the familial ESCC patients in the high-risk northern China may be potentially benefit improved cancer detection. Our study is limited by the moderate sample size and further study with a larger cohort of familial ESCC and control population is warranted for validation to substantiate the findings in the current study. 
Supplementary Materials: The following are available online at http://www.xxxxx, Table S1: Deleterious missense $R A D 50$ variants with CADD score $>=25$. Table S2: The IC 50 of various genotoxic compounds in U2OS overexpressing vector alone (VA), RAD50 ${ }^{\mathrm{WT}}, R A D 50^{\mathrm{Q} 672 \mathrm{X}}$ and $R A D 50^{\mathrm{L} 1264 \mathrm{~F}}$.

Author Contributions Conceptualization, J.M.Y.K. and M.L.L.; methodology, J.M.Y.K. and S.Y.L.; software, S.Y.L. and N.L.; validation, S.Y.L., C.W.Y.W. and A.W.Y.C., L.C.L. and S.S.A.C.; formal analysis, N.L. and S.Y.L.; investigation, S.Y.L.; data curation, L.N.; writing-original draft preparation, J.M.Y.K. and S.Y.L.; writingreview and editing, J.M.Y.K., S.Y.L. N.L. and M.L.L.; visualization, J.M.Y.K. and S.Y.L.; supervision, J.M.Y.K. and M.L.L.; project administration, J.M.Y.K. and M.L.L.; funding acquisition, J.M.Y.K. and M.L.L. All authors have read and agreed to the published version of the manuscript.

Funding: "This research was funded by the Asian Fund for Cancer Research and Collaborative Research Fund (grant number C7031.15G) to M.L.L.; the Seed Fund for Basic Research from the University of Hong Kong (grant number 201611159317) and General Research Fund (grant number 17118517) to J.M.Y.K.

Acknowledgments: The authors acknowledge Prof. Tanya Paull of the University of Texas at Austin, USA for her generosity of providing the RAD50 wildtype construct as a gift.

Conflicts of Interest: The authors declare no conflict of interest. The funders had no role in the design of the study; in the collection, analyses, or interpretation of data; in the writing of the manuscript, or in the decision to publish the results.

\section{References}

1. Bray, F., Ferlay, J., Soerjomataram, I., Siegel, R.L., Torre, L.A., Jemal, A. Global cancer statistics 2018: GLOBOCAN estimates of incidence and mortality worldwide for 36 cancers in 185 countries. CA Cancer J Clin 2018, 68, 394-424. doi: 10.3322/caac.21492

2. Abnet, C.C., Arnold, M., Wei, W.Q. Epidemiology of Esophageal Squamous Cell Carcinoma. Gastroenterology 2018, 154, 360-373. doi: 10.1053/j.gastro.2017.08.023

3. Wang, L.D., Zhou, F.Y., Li, X.M., Sun, L.D., Song, X., Jin, Y., Li, J.M., Kong, G.Q., Qi, H., Cui, J. et al. Genomewide association study of esophageal squamous cell carcinoma in Chinese subjects identifies susceptibility loci at PLCE1 and C20orf54. Nat Genet 2010, 42, 759-763. doi: 10.1038/ng.648

4. Wu, C., Wang, Z., Song, X., Feng, X.S., Abnet, C.C., He, J., Hu, N., Zuo, X.B., Tan, W., Zhan, Q. et al. Joint analysis of three genome-wide association studies of esophageal squamous cell carcinoma in Chinese populations. Nat Genet 2014, 46, 1001-1006. doi: 10.1038/ng.3064

5. Cui, R., Kamatani, Y., Takahashi, A., Usami, M., Hosono, N., Kawaguchi, T., Tsunoda, T., Kamatani, N., Kubo, M., Nakamura, Y. et al. Functional variants in ADH1B and ALDH2 coupled with alcohol and smoking synergistically enhance esophageal cancer risk. Gastroenterology 2009, 137, 1768-1775. doi: 10.1053/j.gastro.2009.07.070

6. Chang-Claude, J., Becher, H., Blettner, M., Qiu, S., Yang, G., Wahrendorf, J. Familial aggregation of oesophageal cancer in a high incidence area in China. Int J Epidemiol 1997, 26, 1159-1165. doi:

7. Hu, N., Dawsey, S.M., Wu, M., Bonney, G.E., He, L.J., Han, X.Y., Fu, M., Taylor, P.R. Familial aggregation of oesophageal cancer in Yangcheng County, Shanxi Province, China. Int J Epidemiol 1992, 21, 877-882. doi:

8. Ko, J.M., Ning, L., Zhao, X.K., Chai, A.W.Y., Lei, L.C., Choi, S.S.A., Tao, L., Law, S., Kwong, A., Lee, N.P. et al. BRCA2 loss-of-function germline mutations are associated with esophageal squamous cell carcinoma risk in Chinese. Int J Cancer 2020, 146, 1042-1051. doi: 10.1002/ijc.32619

9. Van Den Bosch, M., Bree, R.T., Lowndes, N.F. The MRN complex: coordinating and mediating the response to broken chromosomes. EMBO Rep 2003, 4, 844-849. doi: 10.1038/sj.embor.embor925

10. Robison, J.G., Elliott, J., Dixon, K., Oakley, G.G. Replication protein A and the Mre11.Rad50.Nbs1 complex co-localize and interact at sites of stalled replication forks. J Biol Chem 2004, 279, 34802-34810. doi: 
11. Quennet, V., Beucher, A., Barton, O., Takeda, S., Lobrich, M. CtIP and MRN promote non-homologous endjoining of etoposide-induced DNA double-strand breaks in G1. Nucleic Acids Res 2011, 39, 2144-2152. doi: 10.1093/nar/gkq1175

12. Paull, T.T., Lee, J.H. The Mre11/Rad50/Nbs1 complex and its role as a DNA double-strand break sensor for ATM. Cell Cycle 2005, 4, 737-740. doi: 10.4161/cc.4.6.1715

13. Lee, J.H., Paull, T.T. ATM activation by DNA double-strand breaks through the Mre11-Rad50-Nbs1 complex. Science 2005, 308, 551-554. doi: 10.1126/science.1108297

14. Walsh, T., King, M.C. Ten genes for inherited breast cancer. Cancer Cell 2007, 11, 103-105. doi: S15356108(07)00025-6 [pii]10.1016/j.ccr.2007.01.010

15. Bender, C.F., Sikes, M.L., Sullivan, R., Huye, L.E., Le Beau, M.M., Roth, D.B., Mirzoeva, O.K., Oltz, E.M., Petrini, J.H. Cancer predisposition and hematopoietic failure in Rad50(S/S) mice. Genes Dev 2002, 16, $2237-2251$. doi: 10.1101/gad.1007902

16. Abuzeid, W.M., Jiang, X., Shi, G., Wang, H., Paulson, D., Araki, K., Jungreis, D., Carney, J., O'malley, B.W., Jr., Li, D. Molecular disruption of RAD50 sensitizes human tumor cells to cisplatin-based chemotherapy. J Clin Invest 2009, 119, 1974-1985. doi: 10.1172/JCI33816

17. Dai, W., Zheng, H., Cheung, A.K., Tang, C.S., Ko, J.M., Wong, B.W., Leong, M.M., Sham, P.C., Cheung, F., Kwong, D.L. et al. Whole-exome sequencing identifies MST1R as a genetic susceptibility gene in nasopharyngeal carcinoma. Proc Natl Acad Sci U S A 2016, 113, 3317-3322. doi: 10.1073/pnas.1523436113

18. Kircher, M., Witten, D.M., Jain, P., O'roak, B.J., Cooper, G.M., Shendure, J. A general framework for estimating the relative pathogenicity of human genetic variants. Nat Genet 2014, 46, 310-315. doi: 10.1038/ng.2892 19. Chan, S.H., Yee Ko, J.M., Chan, K.W., Chan, Y.P., Tao, Q., Hyytiainen, M., Keski-Oja, J., Law, S., Srivastava, G., Tang, J. et al. The ECM protein LTBP-2 is a suppressor of esophageal squamous cell carcinoma tumor formation but higher tumor expression associates with poor patient outcome. Int J Cancer 2011, 129, 565-573. doi: 10.1002/ijc. 25698

20. Yu, V.Z., Wong, V.C., Dai, W., Ko, J.M., Lam, A.K., Chan, K.W., Samant, R.S., Lung, H.L., Shuen, W.H., Law, S. et al. Nuclear Localization of DNAJB6 Is Associated With Survival of Patients With Esophageal Cancer and Reduces AKT Signaling and Proliferation of Cancer Cells. Gastroenterology 2015, 149, 1825-1836 e1825. doi: 10.1053/j.gastro.2015.08.025

21. Yu, V.Z., Ko, J.M.Y., Ning, L., Dai, W., Law, S., Lung, M.L. Endoplasmic reticulum-localized ECM1b suppresses tumor growth and regulates MYC and MTORC1 through modulating MTORC2 activation in esophageal squamous cell carcinoma. Cancer Lett 2019, 461, 56-64. doi: 10.1016/j.canlet.2019.07.005

22. Ng, H.Y., Ko, J.M., Yu, V.Z., Ip, J.C., Dai, W., Cal, S., Lung, M.L. DESC1, a novel tumor suppressor, sensitizes cells to apoptosis by downregulating the EGFR/AKT pathway in esophageal squamous cell carcinoma. Int J Cancer 2016, 138, 2940-2951. doi: 10.1002/ijc.30034

23. Damiola, F., Pertesi, M., Oliver, J., Le Calvez-Kelm, F., Voegele, C., Young, E.L., Robinot, N., Forey, N., Durand, G., Vallee, M.P. et al. Rare key functional domain missense substitutions in MRE11A, RAD50, and NBN contribute to breast cancer susceptibility: results from a Breast Cancer Family Registry case-control mutationscreening study. Breast Cancer Res 2014, 16, R58. doi: 10.1186/bcr3669

24. Fan, C., Zhang, J., Ouyang, T., Li, J., Wang, T., Fan, Z., Fan, T., Lin, B., Xie, Y. RAD50 germline mutations are associated with poor survival in BRCA1/2-negative breast cancer patients. Int J Cancer 2018. doi: 10.1002/ijc.31579

25. Flores-Perez, A., Rafaelli, L.E., Ramirez-Torres, N., Arechaga-Ocampo, E., Frias, S., Sanchez, S., Marchat, L.A., Hidalgo-Miranda, A., Quintanar-Jurado, V., Rodriguez-Cuevas, S. et al. RAD50 targeting impairs DNA 
damage response and sensitizes human breast cancer cells to cisplatin therapy. Cancer Biol Ther 2014, 15, 777-788. doi: $10.4161 /$ cbt.28551

26. Lajud, S.A., Nagda, D.A., Yamashita, T., Zheng, J., Tanaka, N., Abuzeid, W.M., Civantos, A., Bezpalko, O., O'malley, B.W., Jr., Li, D. Dual disruption of DNA repair and telomere maintenance for the treatment of head and neck cancer. Clin Cancer Res 2014, 20, 6465-6478. doi: 10.1158/1078-0432.CCR-14-0176

27. Gatei, M., Kijas, A.W., Biard, D., Dork, T., Lavin, M.F. RAD50 phosphorylation promotes ATR downstream signaling and DNA restart following replication stress. Hum Mol Genet 2014, 23, 4232-4248. doi: $10.1093 / \mathrm{hmg} / \mathrm{ddu} 141$

28. Heikkinen, K., Rapakko, K., Karppinen, S.M., Erkko, H., Knuutila, S., Lundan, T., Mannermaa, A., Borresen-Dale, A.L., Borg, A., Barkardottir, R.B. et al. RAD50 and NBS1 are breast cancer susceptibility genes associated with genomic instability. Carcinogenesis 2006, 27, 1593-1599. doi: 10.1093/carcin/bgi360

29. Al-Ahmadie, H., Iyer, G., Hohl, M., Asthana, S., Inagaki, A., Schultz, N., Hanrahan, A.J., Scott, S.N., Brannon, A.R., Mcdermott, G.C. et al. Synthetic lethality in ATM-deficient RAD50-mutant tumors underlies outlier response to cancer therapy. Cancer Discov 2014, 4, 1014-1021. doi: 10.1158/2159-8290.CD-14-0380

30. Hopfner, K.P., Craig, L., Moncalian, G., Zinkel, R.A., Usui, T., Owen, B.A., Karcher, A., Henderson, B., Bodmer, J.L., Mcmurray, C.T. et al. The Rad50 zinc-hook is a structure joining Mre11 complexes in DNA recombination and repair. Nature 2002, 418, 562-566. doi: 10.1038/nature00922

(C) 2020 by the authors. Submitted for possible open access publication under the terms and conditions of the Creative Commons Attribution (CC BY) license (http://creativecommons.org/licenses/by/4.0/). 